Editorial

\title{
Neuro-Photobiomodulation: Stimulation of the Brain Using Different Frequencies
}

\author{
Gerhard Litscher*
}

Research Unit for Complementary and Integrative Laser Medicine, Research Unit of Biomedical Engineering in Anesthesia and Intensive Care Medicine, and Traditional Chinese Medicine (TCM) Research Center Graz, Medical University of Graz, 8036 Graz, Austria; E-Mail: gerhard.litscher@medunigraz.at

* Correspondence: Gerhard Litscher; E-Mail: gerhard.litscher@medunigraz.at

Collection: Trends in Acupuncture and Laser Research and Education

OBM Integrative and Complementary Medicine

2021, volume 6, issue 1

doi:10.21926/obm.icm.2101003
Received: January 18, 2021

Accepted: January 18, 2021

Published: January 20, 2021

\begin{abstract}
Neuro-photobiomodulation (PBM) is a relatively novel optical stimulation method that is not yet generally accepted in conventional medicine. Therefore, evidence-based research in this sub-area, which is important for laser medicine, is necessary in order to provide appropriate scientific evidence for a better understanding of neuro-PBM. In this editorial, a summary of the recent results with stimulation using different frequencies (e.g., $40 \mathrm{~Hz}, 136.1 \mathrm{~Hz}$ ) is presented. The contribution is intended to stimulate researchers to continue working in this promising area of neuro-PBM.
\end{abstract}

\section{Keywords}

Neuro-Photobiomodulation; LED (light-emitting diode); light therapy; $40 \mathrm{~Hz}$; OM frequency $136.1 \mathrm{~Hz}$; bispectral index; basic research; integrative and complementary medicine

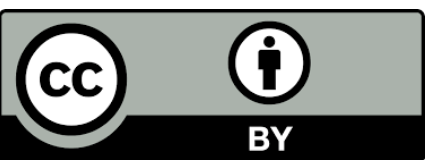

(C) 2021 by the author. This is an open access article distributed under the conditions of the Creative Commons by Attribution License, which permits unrestricted use, distribution, and reproduction in any medium or format, provided the original work is correctly cited. 


\section{Cerebral Photobiomodulation (PBM)}

Cerebral PBM [1], a new treatment option, is still under experimental development. However, it has already been used in different approaches like traumatic brain injury (TBI) and stroke [2] or cerebral ischemia [3, 4], Alzheimer's disease [5], major depressive disorders [6], cognitive rehabilitation [7], and many other related diseases [8].

\section{Frequency-modulated Optical Stimulation}

The most popular method of cerebral PBM involves continuous light stimulation. Several recent studies have shown that certain frequency-modulated optical stimulations could have a very special impact on neuronal functions. Wang et al. (2019) reported that transcranial PBM with 1064-nm laser modulates brain electroencephalogram rhythms [9]. In our experiments, we used light-emitting diode (LED) stimulation instead of light amplification by stimulated emission of radiation (LASER) stimulation [10]. Interestingly, at $40 \mathrm{~Hz}$ frequency and $136.1 \mathrm{~Hz}$ (Om-frequency from meditation), significant changes in electroencephalographic (EEG) activity were noticed. In this context, a study by Hauswald et al. (2015) [11] suggested that during Zen meditation using Om-frequency, highfrequency gamma power in the cingulate cortex and somatosensory cortex positively correlated with the degree of self-reported mindfulness [12]. Our results further highlight the EEG similarities of transcranial LED induced sedation and general anesthesia as assessed by bispectral index (BIS) $[8,10]$. Similar results were reported by Litscher et al. (2004) during different stimulation (acupressure, laser stimulation, etc.) of the Yintang acupoint [13].

laccarino et al. (2016) [14] first reported the positive effects of restoring gamma $(40 \mathrm{~Hz})$ oscillations in the visual cortex of a transgenic mice model in Alzheimer's disease; also optogenetically driving FS-PV-interneurons at gamma $(40 \mathrm{~Hz})$, but not other frequencies, reduced the levels of amyloid- $B(A ß)_{1-40}$ and $A \beta_{1-42}$ isoforms.

Despite several subsequent studies, consensus on using $40 \mathrm{~Hz}$ stimulation for new strategies in the possible treatment of Alzheimer's disease remains elusive. Nevertheless, our experimentally performed BIS measurements showed that at least the stimulation frequency of $40 \mathrm{~Hz}$ could have a (possibly sedative) influence on the bioelectrical function of the brain, which in individual cases can be larger than that compared to continuous stimulation, but does not reach the level of a longerlasting effect with $136.1 \mathrm{~Hz}$ frequency [10]. These preliminary findings are corroborated by the results of the neuromodulatory parameters [10], which were also collected in our experiments. The reduction in the heart rate at a frequency of $136.1 \mathrm{~Hz}$ also showed the soothing effects of this frequency [10]. Our results also indicated that stimulation with $136.1 \mathrm{~Hz}$ frequency had an influence in the 0.1 frequency band in heart rate variability (HRV) [10]. Schwerdtfeger et al. (2020) concluded that the $0.1 \mathrm{~Hz}$ oscillations in the heart and the brain seem to be coupled, thereby indicating central pacemakers on the heart rhythm; breathing at 6 breaths per minute could induce coherence of the $0.1 \mathrm{~Hz}$ oscillations; thus, facilitating physical and psychological function. They stated that central nervous system function modifies the rhythm of the heart and vice versa, suggesting that HRV could be a useful indicator of central-autonomic integration and that $0.1 \mathrm{~Hz}$ oscillations play a major role in physical and mental health via optimizing energy supply [15].

It is highlighted that our preliminary results only present a new PBM design, and the potential of the method should be validated with the first preliminary data in prior studies $[8,10]$. Interestingly, 
the maximum sedative effects were observed with stimulation of $136.1 \mathrm{~Hz}$ (Om frequency) [10]. For this reason, we suggest the scientific community to consider in detail our initial results obtained from our experimental dataset through this editorial in the English language. Possibly, this can initiate further research to bring more clarity to the topic.

\section{Future Aspects}

Neuro-PBM showed that the stimulation with different frequencies resulted in different EEG and neuromodulatory effects. Furthermore, placebo stimulation showed a different effect. This indicates that in addition to continuous stimulation, frequency-related stimulation techniques can also be extremely helpful in improving the performance of neuro-PBM.

This editorial will help in further understanding the novel methods using different frequencies to treat different conditions in years ahead, stimulating further research and motivating the practitioners and researchers to improvise PBM interventions for wider acceptance.

\section{Acknowledgments}

Parts of this editorial have been published in modified form as a report in German language in Akupunktur \& Aurikulomedizin in 2020 [10].

\section{Author Contributions}

Gerhard Litscher did all the research work of this study.

\section{Funding}

TCM Research Center Graz, Medical University of Graz

\section{Competing Interests}

The author does not declare any conflict of interest.

\section{References}

1. Salehpour F, Mahmoudi J, Kamari F, Sadigh-Eteghad S, Rasta SH, Hamblin MR. Brain photobiomodulation therapy: A narrative review. Mol Neurobiol. 2018; 55: 6601-6636.

2. Hamblin MR. Photobiomodulation for traumatic brain injury and stroke. J Neurosci Res. 2018; 96: 731-743.

3. Argibay B, Campos F, Perez-Mato M, Vieites-Prado A, Correa-Paz C, López-Arias E, et al. Lightemitting diode photobiomodulation after cerebral ischemia. Front Neurol. 2019; 10: 911.

4. Wang R, Dong Y, Lu Y, Zhang W, Brann DW, Zhang Q. Photobiomodulation for global cerebral ischemia: Targeting mitochondrial dynamics and functions. Mol Neurobiol. 2019; 56: 1852-1869.

5. Hamblin MR. Photobiomodulation for Alzheimer's disease: Has the light dawned? Photonics. 2019; 6: 77.

6. Cassano P, Petrie SR, Hamblin MR, Henderson TA, losifescu DV. Review of transcranial photobiomodulation for major depressive disorder: Targeting brain metabolism, inflammation, oxidative stress, and neurogenesis. Neurophotonics. 2016; 3: 031404. 
7. Carneiro AM, Poiani GC, Zaninnoto AL, Lazo Osorio R, Oliveira ML, Paiva WS, et al. Transcranial photobiomodulation therapy in the cognitive rehabilitation of patients with cranioencephalic trauma. Photobiomodul Photomed Laser Surg. 2019; 37: 657-666.

8. Litscher G. Transcranial photobiomodulation and related scientific aspects. Graz: DMS; 2021.

9. Wang X, Dmochowski JP, Zeng L, Kallioniemi E, Husain M, Gonzalez-Lima F, Liu H. Transcranial photobiomodulation with 1064-nm laser modulates brain electroencephalogram rhythms. Neurophotonics. 2019; 6: 025013.

10. Litscher G. Transcranial frequency-modulated photobiomodulation. Akupunktur und Aurikulomedizin. 2020: 46: 27-34.

11. Hauswald A, Übelacker T, Leske S, Weisz N. What it means to be Zen: Marked modulations of local and interareal synchronization during open monitoring meditation. Neuroimage. 2015; 108: 265-273.

12. Lee DJ, Kulubya E, Goldin P, Goodarzi A, Girgis F. Review of the neural oscillations underlying meditation. Front Neurosci. 2018; 12: 178.

13. Litscher G. Effects of acupressure, manual acupuncture and Laserneedle acupuncture on EEG bispectral index and spectral edge frequency in healthy volunteers. Eur J Anaesthesiol. 2004; 21: 13-19.

14. laccarino HF, Singer AC, Martorell AJ, Rudenko A, Gao F, Gillingham TZ, et al. Gamma frequency entrainment attenuates amyloid load and modifies microglia. Nature. 2016; 540: 230-235.

15. Schwerdtfeger AR, Schwarz G, Pfurtscheller K, Thayer JF, Jarczok MN, Pfurtscheller G. Heart rate variability (HRV): From brain death to resonance breathing at 6 breaths per minute. Clin Neurophysiol. 2020; 131: 676-693.

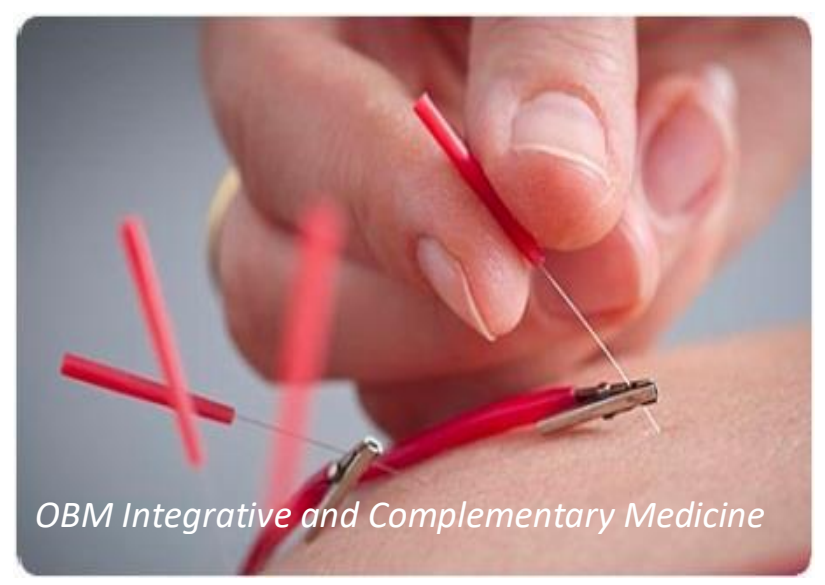

Enjoy OBM Integrative and Complementary Medicine by:

1. Submitting a manuscript

2. Joining in volunteer reviewer bank

3. Joining Editorial Board

4. Guest editing a special issue

For more details, please visit:

http://www.lidsen.com/journals/icm 